\title{
Morphology of Nonmetallic-inclusion Clusters Observed in Molten Metal by X-ray Micro-Computed Tomography (CT)
}

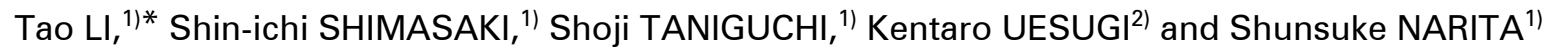 \\ 1) Graduate School of Environmental Studies, Tohoku University, Aoba-yama 6-6-20, Aoba-ku, Sendai, $980-8579$ Japan. \\ 2) Japan Synchrotron Radiation Research Institute (JASRI)/SPring-8, 1-1-1 Kouto, Sayo-cho, Sayo-gun, Hyogo, $679-5198$ Japan.
}

(Received on April 9, 2013; accepted on June 17, 2013)

\begin{abstract}
The removal of non-metallic inclusions in the metallurgical process greatly affects the properties of the final products. The structure of inclusion clusters plays a key role in inclusion behaviors of their removal process, such as coagulation, flotation and bubble adhesion. However, it is rare to find reports quantitatively investigating the morphology of inclusion clusters in metal system. On the other hand, to quantitatively estimate the inclusion clusters in metal, it is required to distinguish clusters on twodimensional (2D) cross-sectional images of the as-polished samples. In this study, $\mathrm{TiB}_{2}$ particle clusters were prepared in a mechanically agitated crucible containing molten $\mathrm{Al}$ at $1073 \mathrm{~K}$. The samples of $\mathrm{Al-TiB_{2 }}$ were measured by X-ray micro-computed tomography (micro-CT) to obtain the three-dimensional (3D) information of $\mathrm{TiB}_{2}$ particles and clusters in solid Al. The images of $3 \mathrm{D}$ particle clusters in solid Al were extracted and reconstructed by self-developed programs. A series of parameters were defined to describe the $3 D$ characteristics of clusters and their 2D cross-sections. The effects of agitation time and speed on the cluster structure were investigated. A program was developed to distinguish clusters in $2 \mathrm{D}$ crosssections through the use of the 3D cluster information (DC-2D-3D) obtained from X-ray micro-CT.
\end{abstract}

KEY WORDS: inclusion coagulation; three-dimensional analysis; morphology of cluster; two-dimensional cluster; X-ray micro-CT.

\section{Introduction}

In steelmaking process, fine inclusion particles in molten steel coagulate each other in turbulent flow and grow up to clusters which are harmful to the properties of steel products. The structure of clusters greatly affects the inclusion behaviors in molten metal, such as coagulation, flotation, and bubble adhesion, which play key roles in inclusion removal. The three-dimensional (3D) analysis of the structure of inclusion clusters will provide significant information for understanding the mechanisms of inclusion cluster behaviors in liquid metal relating to cleanup of steel products. Many research works have been done focusing the mechanism of particle coagulation process. ${ }^{1-6)}$ However, in most particle coagulation models, the clusters formed by particle coagulation are assumed to be spheres because of the experimental difficulties to get the 3D information of these clusters as well as the theoretical difficulties. On the other hand, to evaluate and improve the process of steel making, quantitative estimation of the inclusion clusters in solid metal is indispensible, which is traditionally performed on twodimensional (2D) cross-sections of as-polished samples. The obstacle of the 2D estimation is to distinguish clusters on the cross-sectional images, on which each cluster appears as a few of closed but isolated particles. If the $3 \mathrm{D}$ informa-

* Corresponding author: E-mail: litao0518@hotmail.com DOI: http://dx.doi.org/10.2355/isijinternational.53.1943 tion of clusters in metal is available, it might be applied to distinguishing clusters in 2D cross-sectional images.

Fractal dimension ${ }^{7-9)}$ was introduced to describe the cluster structure that indicates the relationship between the volume (area) and the size in length of a cluster. Some research works have been performed to derive the cluster structure using fractal dimension. ${ }^{10-19)}$ Jullien et al. ${ }^{10)}$ investigated the fractal dimension of clusters by the diffusion limited aggregation (DLA) model, ${ }^{11-14)}$ and it was shown to be $1.78 \pm 0.05$ for 2D clusters. Tozawa et al. ${ }^{15)}$ firstly reported the fractal dimension of alumina clusters in the steel sample as 1.8 estimated from the 2D cross-sectional measurement. Doo et al. ${ }^{16)}$ investigated the 2D and 3D fractal dimension of the alumina cluster extracted from steel according to the projected area of the cluster calculated by Forrest method. ${ }^{17)}$ It reported the 2D fractal dimension is in the range of 1.80-1.95. And the 3D fractal dimension was estimated following a relation between 2D and 3D fractal dimension of clusters given by Lee and $\mathrm{Kramer}^{18)}$ and fell into a range of 1.882.07. Eggersdorfer et al. ${ }^{19)}$ simulated fragmentation and restructuring of soft-agglomerates under shear flow by discrete element method (DEM). A cluster with fractal dimension of about 1.8 starts to be stretched toward a linear shape, and then the child clusters relax until a steady state with fractal dimension about $2.03 \pm 0.2$. However, it is very difficult to find the experimental reports about $3 \mathrm{D}$ cluster structure analysis, especially the non-metallic particle clusters in metal system because of its opaqueness and high melting point. 
Recently, some 3D analyses of the pore and non-metallic particles in metal were made by X-ray micro-computed tomography (micro-CT) ${ }^{20-28)}$ which is well known as nondestructive inspection method. In this study, $\mathrm{TiB}_{2}$ particle clusters were prepared in a mechanically agitated crucible containing molten $\mathrm{Al}$ and $\mathrm{TiB}_{2}$ particles. A series of sliced $2 \mathrm{D}$ cross-sectional images of $\mathrm{Al}_{-} \mathrm{TiB}_{2}$ samples were obtained by X-ray micro CT available in SPring- 8 , which is a large synchrotron radiation facility which delivers the most powerful synchrotron radiation currently available in Japan. 3D $\mathrm{TiB}_{2}$ particles and clusters were extracted and reconstructed from $2 \mathrm{D}$ image stacks by a self-developed program named 3D Extractor based on ImageJ, a public domain image processing software. A number of parameters were defined to describe the characteristics of $3 \mathrm{D}$ clusters and their 2D cross-sections. The influence of the agitation time and speed of impeller on the cluster structure are discussed. A program, DC-2D-3D (distinguish clusters on 2D cross-sections based on 3D information), was developed to distinguish clusters on cross-sectional images. Criteria for determining 2D cross-sectional clusters were obtained by analyzing 3D cluster information. The reliability of the program is validated using the $3 \mathrm{D}$ information of clusters in the image stacks.

\section{2. $\mathbf{T i B}_{2}$ Clusters in Molten $\mathbf{A l}$}

\subsection{Materials}

Figure 1 shows the SEM image of $\mathrm{TiB}_{2}$ particles used to prepare clusters in the turbulent flow of molten Al. Figure 2 shows the $\mathrm{TiB}_{2}$ particle size distribution (PSD) measured by Coulter Counter-3. Particles with equivalent diameter less than $2 \mu \mathrm{m}$ were ignored because of the limitation of Coulter Counter-3. Figure 2(a) shows the PSD by number

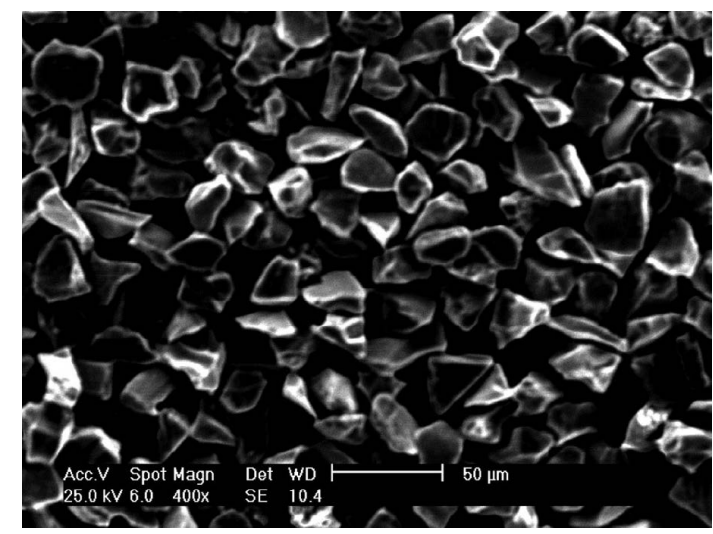

Fig. 1. SEM image of $\mathrm{TiB}_{2}$ particles used to prepare clusters in molten Al.

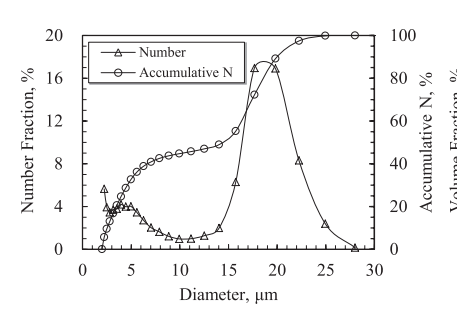

(a)

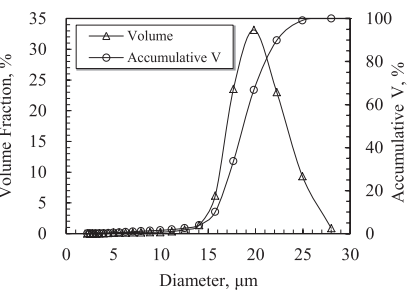

(b)
Fig. 2. $\mathrm{TiB}_{2}$ particle size distribution by Coulter Counter-3. and accumulative number fractions, and peaks of the PSD appear at both about $5 \mu \mathrm{m}$ and $20 \mu \mathrm{m}$ by number fraction, which is corresponding to the change of slope on the PSD by accumulative number fraction. Whereas the peaks in the range of small particles disappear in the PSD by volume and accumulative volume fractions in Fig. 2(b), because small particles contribute little to the total particle volume.

\subsection{Experimental Procedures}

Figure 3 shows the experimental apparatus to prepare $\mathrm{TiB}_{2}$ clusters in molten $\mathrm{Al}$. $\mathrm{TiB}_{2}$ particles included in semisolid slurry ${ }^{28)}$ of Al-7wt.\%Si alloy $(878 \mathrm{~K}$ ) were mixed into molten aluminum at $1123 \mathrm{~K}$ in a crucible $\left(31 \% \mathrm{Al}_{2} \mathrm{O}_{3}-\right.$ $69 \% \mathrm{SiO}_{2}$ ). An alumina-coated two-paddle impeller was introduced to agitate and generate turbulent flow in the crucible with two baffle plates. The temperature of the molten $\mathrm{Al}$ including particle was kept at about $1073 \mathrm{~K}$. Particles in the molten Al coagulate each other under turbulent flow and form into clusters; the breakup of clusters was occurring at the same time by the shear force in turbulent flow. Samples were sucked from the molten Al by a silica tube of $3.6 \mathrm{~mm}$ ID during the agitation, and quenched by water. Table 1 shows the experimental conditions. Samples were taken in each experiment with agitation time at 1, 3, 6, 12, 25, 60 and $105 \mathrm{~min}$ and labeled as, for example, Exp. B-3 is from experiment B after 3 min agitation.

\section{Reconstruction of Three-Dimensional Cluster Image}

\subsection{X-ray Micro-CT}

The samples taken from molten Al including clusters were mechanically processed into cylinders that were 0.8 $\mathrm{mm}$ in diameter and $2-5 \mathrm{~mm}$ long. The samples were measured by the X-ray micro-CT system installed in the beamline BL20XU at SPring-8, which is with an energy of 19.99

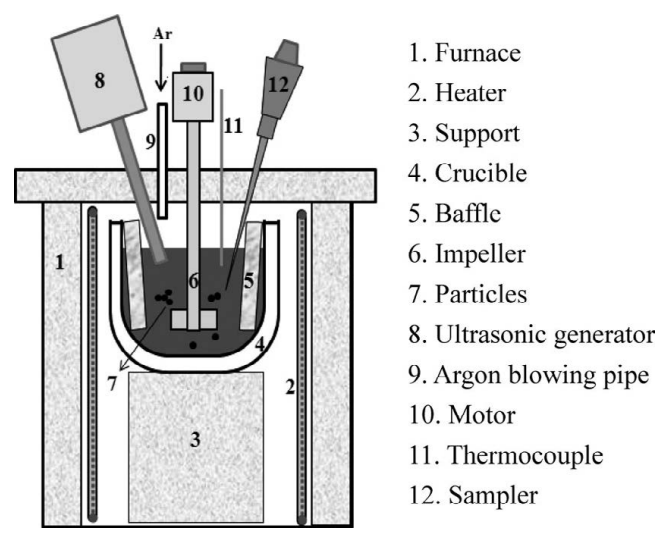

Fig. 3. Experimental apparatus to prepare $\mathrm{TiB}_{2}$ clusters in molten Al.

Table 1. Experimental conditions to prepare $\mathrm{TiB}_{2}$ clusters in molten $\mathrm{Al}$.

\begin{tabular}{lccc}
\hline & Exp. A & Exp. B & Exp. C \\
\hline Temperature & \multicolumn{3}{c}{ about 1073 K } \\
Agitation speed (rpm) & 300 & 400 & 500 \\
Total weight (Al-TiB 2 ) (g) & 415 & 413 & 398 \\
TiB $_{2}$ volume fraction (\%) & 0.22 & 0.54 & 0.43 \\
\hline
\end{tabular}


$\mathrm{keV}$. Totally 1439 sliced (with interval of $0.5 \mu \mathrm{m}$ ) images with a $3 \mathrm{D}$ resolution of $0.5 \mu \mathrm{m} /$ pixel (voxel: $0.125 \mu \mathrm{m}^{3}$ ) from one sample were obtained. Figure 4 shows the principle of X-ray micro-CT.

\subsection{Reconstruction of 3D Cluster Image}

A program named 3D Extractor was developed to extract particles and clusters from an image stack including a series of cross-sectional images (1 439 slices). Figure 5 shows the main flow chart of the program 3D Extractor. The program flow is expressed as follows:

(1) An image stack is read into the program;

(2) For all the particles in 2D slices, the area, perimeter, Feret's diameter in each coordinate (X and $\mathrm{Y}$ ), center position, sequence number in the whole image stack, and the slice number indicating the coordinates $\mathrm{Z}$ values are analyzed.

(3) Step (4) for each image slice $S$ is performed until $S=S_{\max }$, where $S_{\max }$ is the total image slice number in the stack.

(4) When slice $S=1$ (the first slice), all the particles in the present slice are given a new group number indicating the cluster sequence number in the image stack and then step (5) is repeated; For other slices $S>1$, step (5) is repeated.

(5) For each particle $i$ in the present image slice $S$, (6) is repeated until all the particles in the present slice are checked.

(6) Particle $j$ in the slice $S+1$ is checked; Particle $i$ and $j$ are recognized as belonging to one cluster if particle $i$ and $j$ overlap in their projections in the X-Y plane; Particle $j$ is labeled by the same cluster sequence number as particle $i$.

The module A shown in Fig. 5 is used in the case of several particles (e.g. $i=N_{1}, N_{2}, N_{3}, \ldots$ ) in the present slice $S$ overlapped with the same particle $j$ in the slice $S+1$, which is much more complex than single particle overlap because of branch structure of the cluster. In this case, all the particles directly or indirectly connected to particles $i=N_{1}, N_{2}, N_{3}, \ldots$ in the slices before and in the present slice $S$ should be

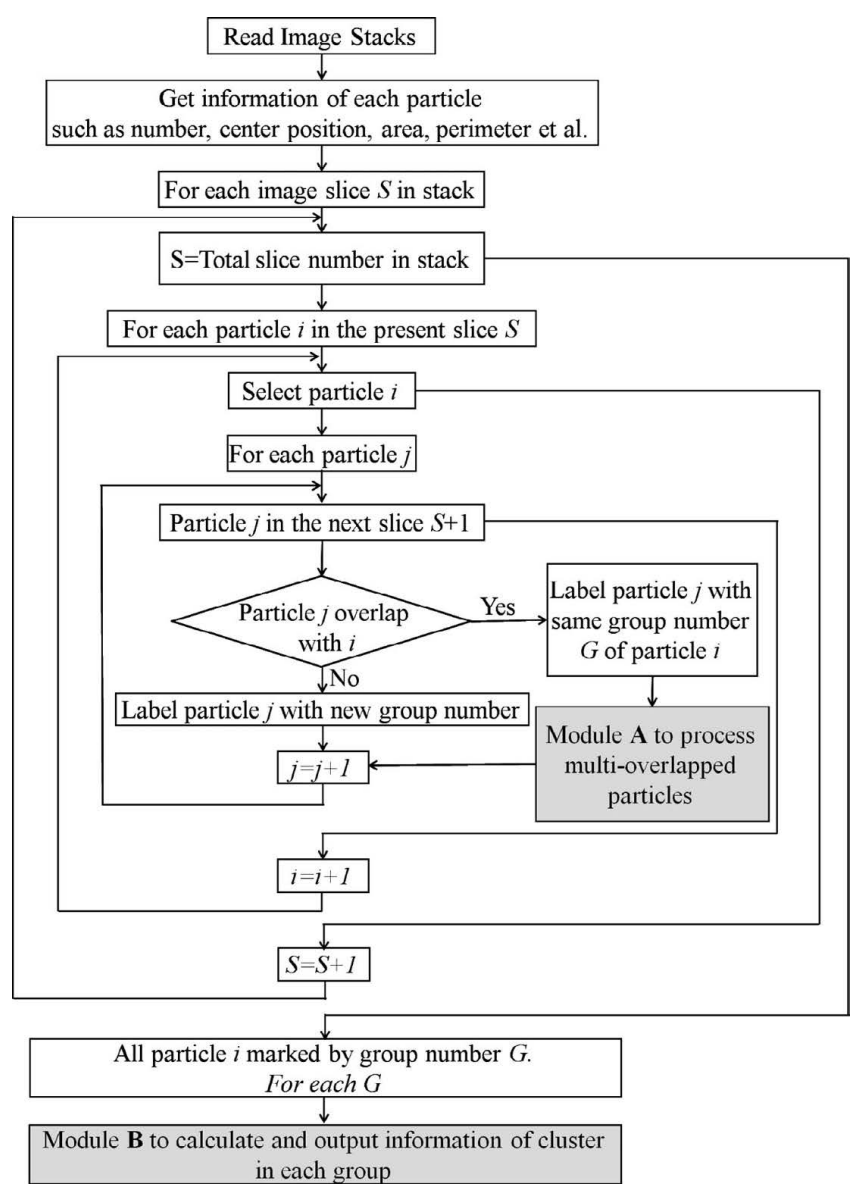

Fig. 5. Program flow chart of 3D Extractor to extract clusters from an image stack.

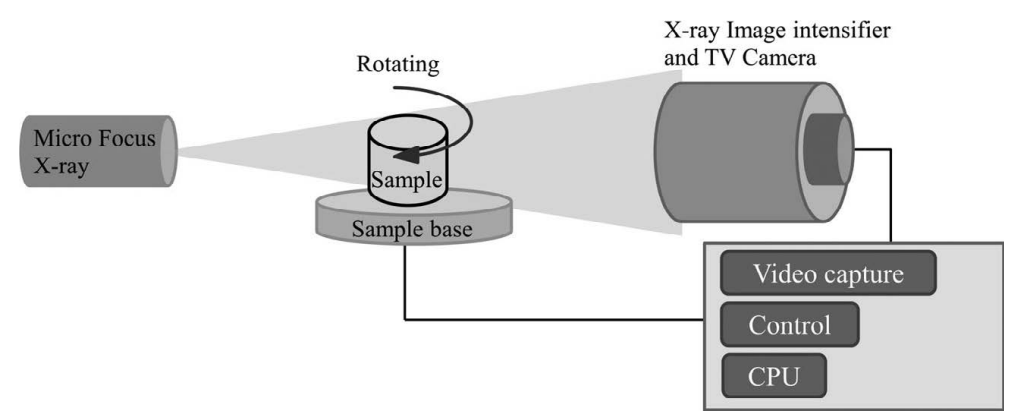

Fig. 4. Principle of X-ray micro-CT.

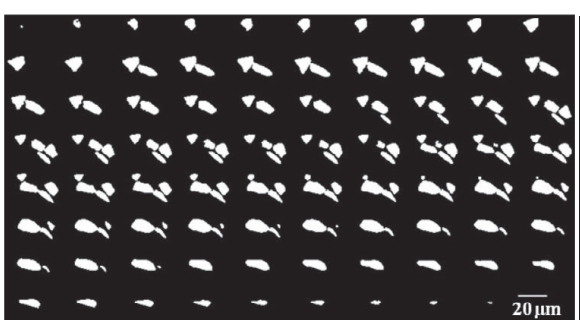

(a) Cross-sectional slices of an extracted cluster.

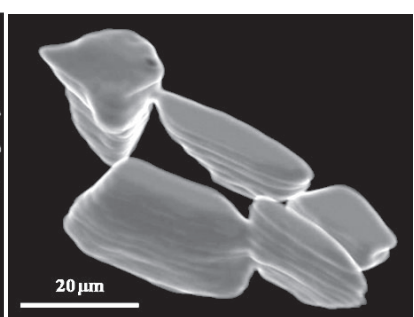

(b) Volume rendering by Osirix.

Fig. 6. Slices of an extracted cluster and $3 \mathrm{D}$ reconstruction. 
Table 2. Parameters to describe $3 \mathrm{D}$ size and structure of particles and clusters.

\begin{tabular}{|c|c|c|}
\hline Type & Parameters & Definition \\
\hline \multirow{8}{*}{ Size } & $d_{x}$ & Feret's diameter in coordinate $\mathrm{X}$ \\
\hline & $d_{y}$ & Feret's diameter in coordinate $\mathrm{Y}$ \\
\hline & $d_{z}$ & Feret's diameter in coordinate $\mathrm{Z}$ \\
\hline & $d_{\text {ave }}$ & Average diameter, $d_{a v e}=\left(d_{x}+d_{y}+d_{z}\right) / 3$ \\
\hline & $d_{\max }$ & Maximum diameter, $d_{\max }=\operatorname{maximum}\left(d_{x}, d_{y}, d_{z}\right)$ \\
\hline & $V_{b o x}$ & Volume of enfolding cube, $V_{b o x}=d_{x} d_{y} d_{z}$ \\
\hline & $V$ & Volume of the cluster \\
\hline & Sur & Surface area of the cluster \\
\hline \multirow{5}{*}{ Structure } & $N_{s, \max , 3 \mathrm{D}}$ & $\begin{array}{l}\text { Maximum particle number in the cross-sections } \\
\text { of a cluster }\end{array}$ \\
\hline & $D_{f, a v e, 3 \mathrm{D}}$ & Fractal dimension by $d_{\text {ave }}, V=\frac{\pi}{6} d_{\text {ave }}^{D_{f, a v e, 3 \mathrm{D}}}$ \\
\hline & $D_{f, \max , 3 \mathrm{D}}$ & Fractal dimension by $d_{\max }, V=\frac{\pi}{6} d_{\max }^{D_{f, \max , 3 \mathrm{D}}}$ \\
\hline & $S_{v}$ & Volumetric specific surface, $S_{v}=S u r / V$ \\
\hline & $\mathrm{Sol}_{v}$ & Volumetric solidity, $S o l_{v}=V / V_{b o x}$ \\
\hline
\end{tabular}

labeled by the same cluster sequence number to particle $j$. The module B shown in Fig. 5 is for calculating the parameters to describe the characteristics of clusters and their cross-sections, which are discussed below.

Figure 6(a) shows the cross-sectional slices of a cluster extracted from an image stack by the program of 3D Extractor. Figure 6(b) shows 3D image of the cluster reconstructed by commercial software, Osirix from the cross-sectional slices shown in Fig. 6(a). Table 2 defines various parameters to describe 3D size and structure of each particle and cluster, which are calculated in the program of 3D Extractor.

\section{3D Characteristics of Particles and Clusters}

\subsection{Statistical Analysis Method}

The samples taken from the experiments were measured by the X-ray micro-CT to generate a series of cross-sectional images for each sample. The particles and clusters were extracted from the image stacks by the program of 3D Extractor. Finite-sample distribution (FSD) ${ }^{29)}$ was introduced, which indicates the probability distribution of statistic parameters based on a random sample system. Figure 7 shows the FSD of the parameters for the clusters from sample Exp. B-6, such as $D_{f, a v e, 3 \mathrm{D}}$ and $D_{f, \max , 3 \mathrm{D}}$ which are used to describe the $3 \mathrm{D}$ structure of the clusters. In order to reduce the artifacts in X-ray micro-CT images, such as dots and lines, the data from 2.5 th to 97.5 th percentile of the FSD for each parameter was used for analysis. The utilized data was specified by the vertical broken lines and the values in Fig. 7. The mode and average values of each parameter marked in the figures were obtained from the FSD of these parameters.

\subsection{Effect of Agitation Time on 3D Parameters of Clus- ters}

In turbulent flow, particle coagulation and breakup are occurring simultaneously during agitation. Therefore, it is necessary to investigate the influence of the agitation time
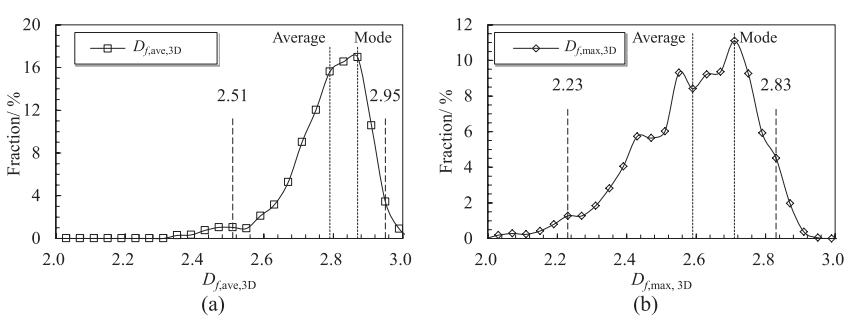

Fig. 7. Value distribution of $3 \mathrm{D}$ parameters of clusters from sample Exp. B-6.

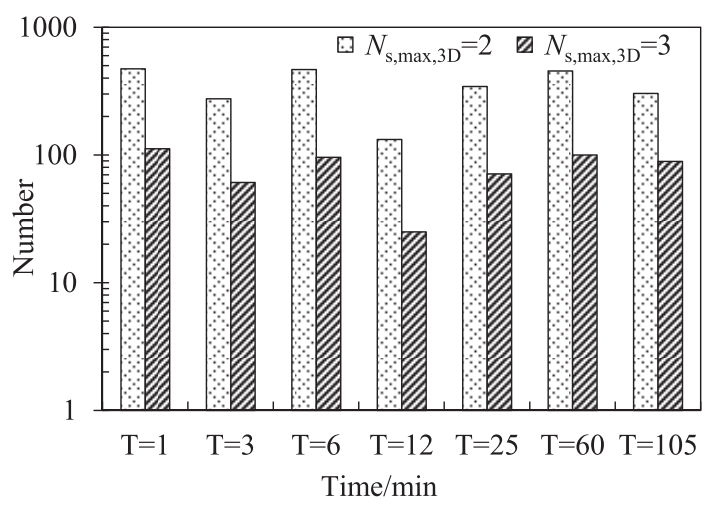

Fig. 8. Number of clusters with same $N_{s, \max , 3 \mathrm{D}}$ collected from Exp. B.

on the cluster structure. Figure 8 shows the number of clusters with $N_{s, \max , 3 \mathrm{D}}=2$ and 3 respectively which are collected from the samples taken in Exp. B at each sampling time. Figures 9 and $\mathbf{1 0}$ show the changes of 3D cluster structure in Exp. B with agitation time for cluster with $N_{s, \max , 3 \mathrm{D}}=2$ and 3 respectively. It is seen from Figs. 9 and 10 that the parameters for 3D cluster structure do not greatly change during the particle coagulation process. Therefore, the agitation time does not affect greatly on the cluster structure. To improve the reliability of statistics for cluster structure, all the clusters with the same $N_{s, \max , 3 \mathrm{D}}$ were collected from all the samples in Exp. B.

\subsection{Change of 3D Clusters Structure with Particle Number}

The particles and clusters collected from Exp. B were divided into groups according to their $N_{s, \max , 3 \mathrm{D}}$ that indicates the maximum particle number in the cross-sections of the particle or cluster. Figure 11 shows the FSD of the parameters for particle or cluster structure according to $N_{s, \max , 3 \mathrm{D} \text {. }}$ There is not apparent changes for $D_{f, \text { ave,3D }}$ and $D_{f, \text { max,3D }}$ with

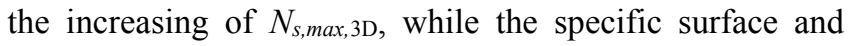
solidity decrease much with the increase of particle number. The decrease in the specific surface is attributed to the increase of the interfaces among particles due to coagulation when the particle number increases. The decrease in solidity, which means the filling rate of a cluster in its covered box, indicates the clusters become looser with the increase of particle number. It can be roughly concluded that the fractal dimension of the $\mathrm{TiB}_{2}$ clusters formed in molten $\mathrm{Al}$ is around 2.7, which is selected as the representative value.

As shown in Fig. 11, the variation of $D_{f, \max , 3 \mathrm{D}}$ is always

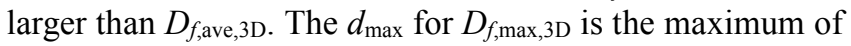
$d_{\mathrm{x}}, d_{\mathrm{y}}$, and $d_{\mathrm{z}}$ which is greatly dependent on the orientation 
ISIJ International, Vol. 53 (2013), No. 11

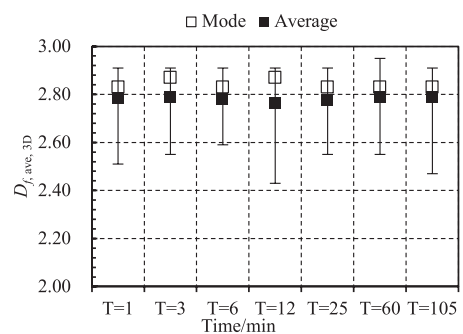

(a)

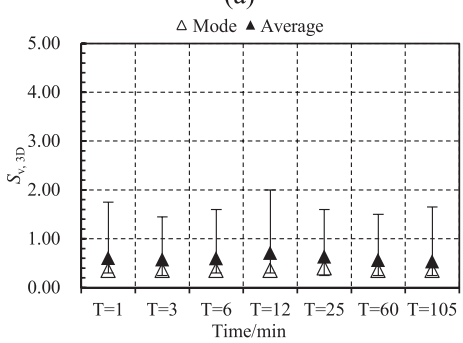

(c)

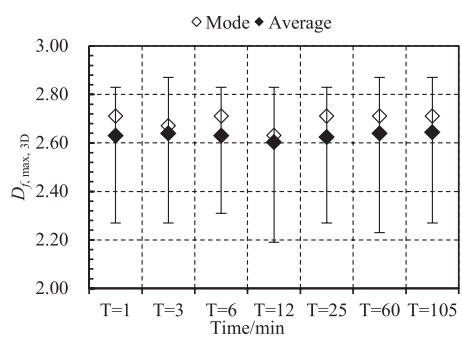

(b)

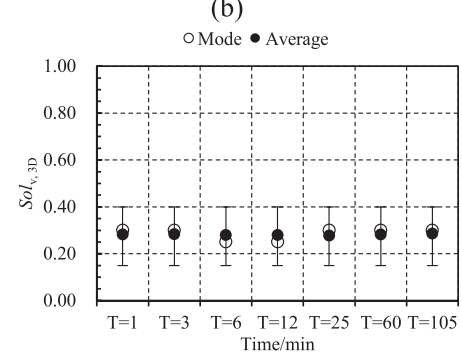

(d)

Fig. 9. Changes of 3D structure of clusters with agitation time.

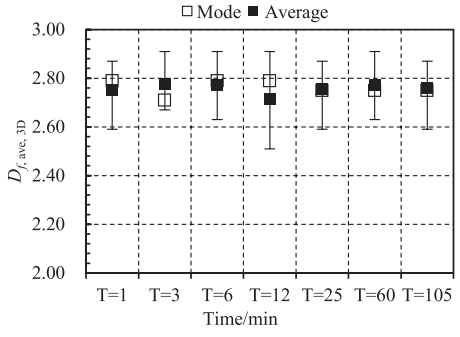

(a)

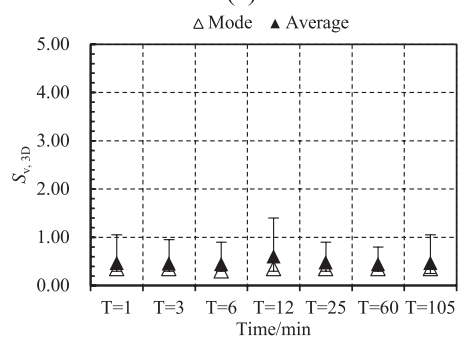

(c)

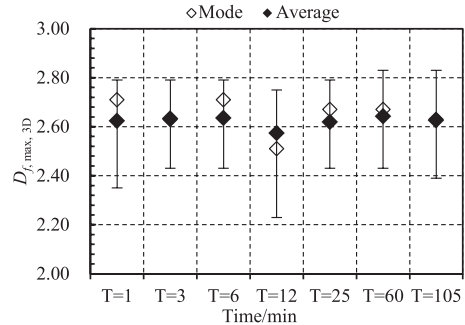

(b)

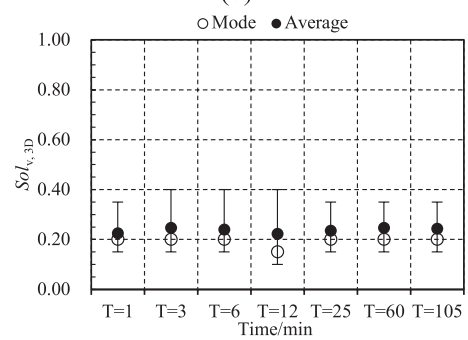

(d)

Fig. 10. Changes of $3 D$ structure of clusters with agitation time.

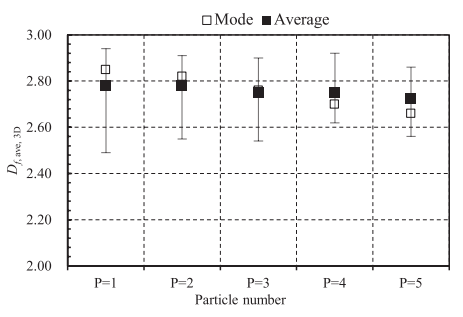

(a)

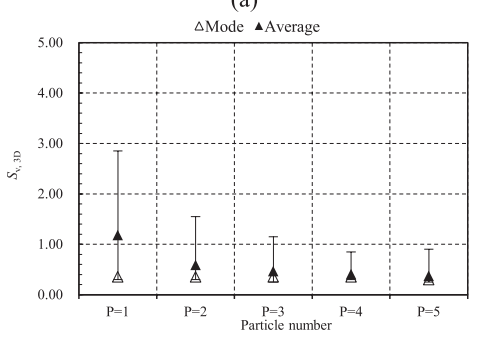

(c)

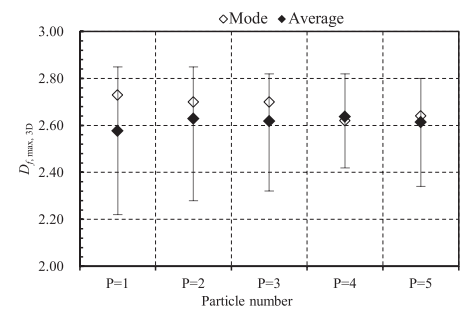

(b)

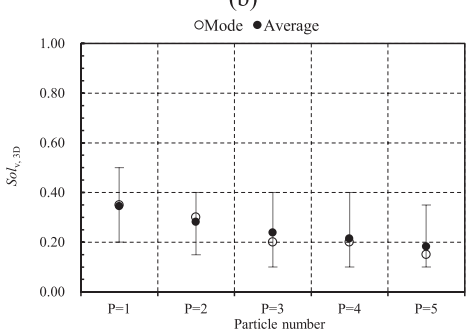

(d)

Fig. 11. Changes of parameters for $3 \mathrm{D}$ cluster structure with $N_{s, \max , 3 \mathrm{D} \text {. }}$ 
of clusters in the solid metal samples. Whereas, the $d_{\text {ave }}$ for calculating $D_{f, \text { ave,3D }}$ is the average of $d_{\mathrm{x}}, d_{\mathrm{y}}$, and $d_{\mathrm{z}}$, thus influence of cluster orientation is reduced. Therefore, the variation of $d_{\mathrm{ave}}$ is much smaller than $d_{\max }$. Figure 12 compares the $d_{\text {ave }}$ and $d_{\max }$ for clusters with various particle number. The ranges of $d_{\max }$ are always larger than those of $d_{\text {ave; }}$; and the average values of $d_{\max }$ is also larger than that of $d_{\text {ave }}$.

\subsection{Influence of Agitation Speed to 3D Clusters Struc- ture}

To investigate the effect of agitation speed on the cluster structure, particles and clusters from the samples of each

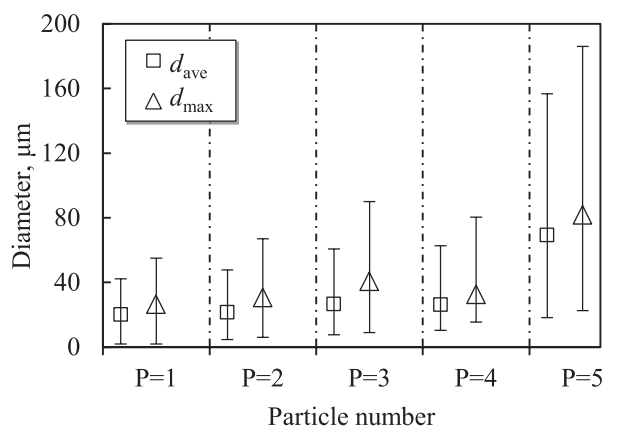

Fig. 12. Comparison of $d_{\text {ave }}$ and $d_{\max }$ for clusters.

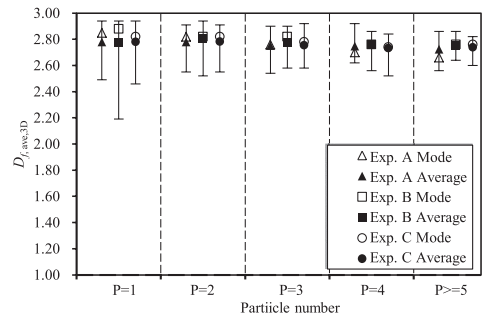

(a)

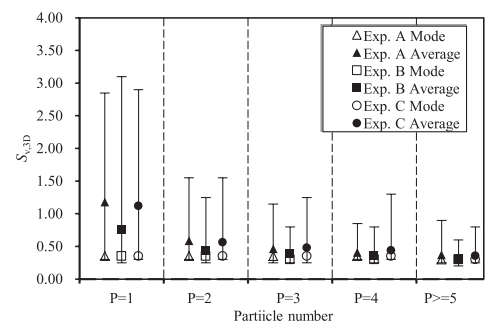

(c)

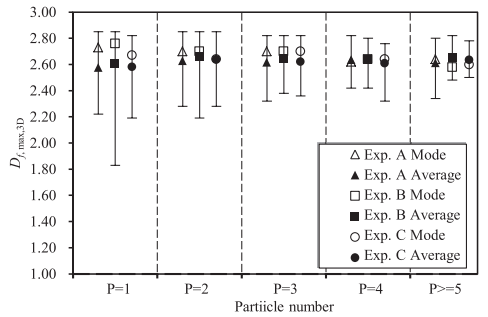

(b)

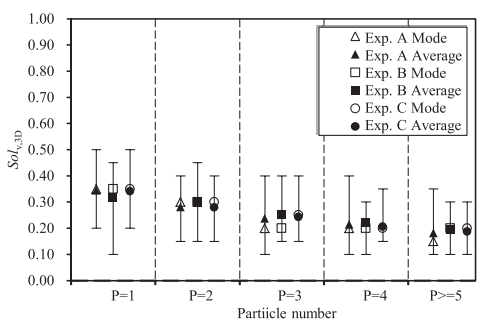

(d)

Fig. 13. Change with agitation speed in FSD of 3D parameters.

Table 3. Examples of $3 \mathrm{D}$ reconstructed $\mathrm{TiB}_{2}$ clusters with parameters.

\begin{tabular}{|c|c|c|c|c|c|c|c|}
\hline \multicolumn{4}{|c|}{ 3D Structure } & \multicolumn{4}{|c|}{ 2D Structure } \\
\hline$N_{s, \max , 3 \mathrm{D}}$ & Parameters & Value & Image & Parameters & Center & $N_{s, \max , 3 \mathrm{D}}$ & Slices of Center and $N_{s, \max , 2 \mathrm{D}}$ \\
\hline \multirow{6}{*}{1} & $D_{\text {f,ave,3D }}$ & 2.79 & & Position & $30 / 60$ & $21 / 60$ & Center \\
\hline & & & & $N_{s, 2 \mathrm{D}}$ & 1 & 1 & \\
\hline & $D_{f, \max , 3 \mathrm{D}}$ & 2.7 & & $D_{f, a v e, 2 \mathrm{D}}$ & 1.95 & 1.91 & \\
\hline & $S_{v}$ & 0.3 & & $D_{f, a v e, 2 \mathrm{D}}$ & 1.89 & 1.9 & $N_{\mathrm{s}, \max , 3 \mathrm{D}}$ \\
\hline & & & & Cir & 0.68 & 0.6 & \\
\hline & $\mathrm{Sol}_{v}$ & 0.23 & $10 \mu \mathrm{m}$ & $\mathrm{Sol}_{s}$ & 0.65 & 0.56 & \\
\hline \multirow{6}{*}{3} & $D_{f, a v e, 3 \mathrm{D}}$ & 2.79 & & Position & $22 / 44$ & $31 / 44$ & Center \\
\hline & & & & $N_{S, 2 \mathrm{D}}$ & 2 & 3 & \\
\hline & $D_{f, \max , 3 \mathrm{D}}$ & 2.59 & & $D_{f, a v e, 2 \mathrm{D}}$ & 1.92 & 1.84 & \\
\hline & $S_{v}$ & 0.27 & & $D_{f, a v e, 2 \mathrm{D}}$ & 1.83 & 1.83 & $N_{\mathrm{s}, \mathrm{max}, 3 \mathrm{D}}$ \\
\hline & & & & Cir & 0.29 & 0.27 & \\
\hline & $\mathrm{Sol}_{v}$ & 0.25 & $10 \mu \mathrm{m}$ & $\mathrm{Sol}_{s}$ & 0.59 & 0.39 & \\
\hline \multirow{6}{*}{7} & $D_{f, a v e, 3 \mathrm{D}}$ & 2.74 & & Position & $50 / 99$ & $29 / 99$ & Center \\
\hline & & & & $N_{s, 2 \mathrm{D}}$ & 4 & 7 & \\
\hline & $D_{f, \max , 3 \mathrm{D}}$ & 2.67 & & $D_{f, a v e, 2 \mathrm{D}}$ & 1.83 & 1.82 & \\
\hline & $S_{v}$ & 0.31 & & $D_{f, a v e, 2 \mathrm{D}}$ & 1.79 & 1.79 & $N_{\mathrm{s}, \max , 3 \mathrm{D}}$ \\
\hline & & & & Cir & 0.12 & 0.12 & \\
\hline & $\mathrm{Sol}_{v}$ & 0.16 & 10 & $\mathrm{Sol}_{s}$ & 0.36 & 0.33 & \\
\hline
\end{tabular}


experiment were collected together and then divided into groups according to $N_{s, \max 3 \mathrm{D}}$ of clusters. Figure 13 compares the parameters of 3D particle and cluster structure from Exp. A, Exp. B, and Exp. C, which have different agitation speeds. It can be seen from Fig. 13 that the parameters are almost same for a certain group with the same $N_{s, \max , 3 \mathrm{D}}$ in the cases of different agitation speeds. It indicates that the 3D parameters for the cluster structure do not change with the agitation speed during the coagulation process, but change somewhat with the $N_{s, \max 3 \mathrm{D}}$ The effects of particle number on the parameters for the 3D cluster structure have different intensity depending on the definition of the parameters. The changes of $S_{\mathrm{v}}$ and $S o l_{v}$, for example, are more sensitive to the increase of $N_{s, \max , 3 \mathrm{D}}$ comparing with the fractal dimensions.

\subsection{D Reconstructed $\mathrm{TiB}_{2}$ Cluster Image}

The 2D slices of $\mathrm{TiB}_{2}$ clusters were extracted from X-ray micro-CT image stacks by program of 3D extractor. The 3D images of these clusters were reconstructed by the commercial software Osirix. Table 3 shows the examples of 3D reconstructed $\mathrm{TiB}_{2}$ clusters with their parameters. The center slice and the slice with particle number same as $N_{s, \max , 3 \mathrm{D}}$ for the cluster are selected for each cluster as examples; the position of these slices are shown by (Sequence number of slice)/(Total slice number of the cluster).

\section{Distinguishing Clusters in 2D Cross-sections}

\subsection{Characteristics of $2 \mathrm{D}$ Cluster}

To obtain the actual cluster number on 2D cross-sectional images, the first and most important step is to distinguish clusters in 2D cross-sections, which are shown as several unconnected particles. A great number of $2 \mathrm{D}$ slices of the $\mathrm{TiB}_{2}$ clusters were obtained from the X-ray micro-CT images by the self-developed program, which has been verified by 3D information in images stacks. Table 4 defines parameters to describe characteristics of 2D cross-sections of clusters, in which the parameters for the 2D cluster structure are very important to distinguish them.

The following procedure is developed to distinguish clusters in 2D cross-sectional images based on the statistical critical ranges of 2D parameters of structure shown in Table 4. In order to determine the criteria of cluster parameters for distinguishing whether a selected group of particles is cluster, both the particle size and number of actual cluster are investigated thoroughly like in Table 5. In the Table, the 2D particles with circular equivalent diameters larger than $10 \mu \mathrm{m}$ were recognized as large particles and others were small ones (L and $\mathrm{S}$, in Table 5). Figure 14 shows the statistics of the characteristics of parameters describing 2D cluster structure according to Table 5, which is derived from the 3D information of actual clusters including thousands of $2 \mathrm{D}$ cluster slices. The critical range for each type of $2 \mathrm{D}$ cluster in Table 5 can be seen in Fig. 14, which indicates the selected particles are one cluster if their parameters fall in this range. It can be roughly derived that the fractal dimension of the 2D cluster slices are around 1.8 according to Fig. 14 , which is selected as the representative value.

\subsection{Program to Distinguish 2D Cluster}

The program of DC-2D-3D (distinguish clusters in 2D cross-sections based on 3D information) was developed by introducing parameters from actual 3D cluster information to distinguish clusters in 2D cross-sectional images. Figure 15 shows an example of the process to distinguish clusters (Model A). Figure 15(A-0) is an original image including particles and clusters. To simplify the explanation, the 2D particle cross-sections are assumed to be mono sized circles. Figure 16 shows the change of 2D parameters with steps in Model A. The process of the program to distinguish 2D clusters is as follows:

(1) A particle is selected (particle with black edge in Fig. 15(A-1)).

(2) A circle is drawn with radius $R$ whose center is at the

Table 4. Parameters to describe characteristics of $2 \mathrm{D}$ cluster crosssections.

\begin{tabular}{|c|c|c|}
\hline Type & Parameters & Definition \\
\hline \multirow{7}{*}{ Size } & $d_{x}$ & Feret's diameter in coordinate $\mathrm{X}$ \\
\hline & $d_{y}$ & Feret's diameter in coordinate $\mathrm{Y}$ \\
\hline & $d_{\text {ave }}$ & Average diameter, $d_{\text {ave }}=\left(d_{x}+d_{y}\right) / 2$ \\
\hline & $d_{\max }$ & Maximum diameter \\
\hline & $S_{\text {rec }}$ & Area of enfolding rectangle, $S_{r e c}=d_{x} d_{y}$ \\
\hline & $S$ & Area of $2 \mathrm{D}$ cross-section \\
\hline & Peri & Perimeter of the cross-section \\
\hline \multirow{5}{*}{ Structure } & $N_{s, 2 \mathrm{D}}$ & Number of particles in all cross-section slices \\
\hline & $D_{f, a v e, 2 \mathrm{D}}$ & Fractal dimension by $d_{\text {ave }}, S=\frac{\pi}{4} d_{\text {ave }}^{D_{f, a v e, 2 \mathrm{D}}}$ \\
\hline & $D_{f, \max , 2 \mathrm{D}}$ & Fractal dimension by $d_{\max }, S=\frac{\pi}{4} d_{\max }^{D_{f, \max , 2 \mathrm{D}}}$ \\
\hline & Cir & Circularity of the cross-section, $\mathrm{Cir}=4 \pi \frac{S}{\mathrm{Peri}^{2}}$ \\
\hline & $\mathrm{Sol}_{s}$ & Area solidity, $S_{0 l}=S / S_{\text {rec }}$ \\
\hline
\end{tabular}

Table 5. Types of 2D clusters according particle size and number.

\begin{tabular}{|c|c|c|c|c|c|c|c|c|}
\hline Particle Number & Type & Size & Particle Number & Type & Size & Particle Number & Type & Size \\
\hline \multirow{2}{*}{1} & $1-\mathrm{A}$ & $0 \mathrm{~L}+1 \mathrm{~S}$ & \multirow{2}{*}{3} & $3-\mathrm{C}$ & $2 \mathrm{~L}+1 \mathrm{~S}$ & \multirow{7}{*}{$\begin{array}{c}n \\
(\mathrm{n} \geq 5, \mathrm{x}>5)\end{array}$} & $5-\mathrm{A}$ & $0 \mathrm{~L}+n \mathrm{~S}$ \\
\hline & $1-B$ & $1 \mathrm{~L}+0 \mathrm{~S}$ & & $3-\mathrm{D}$ & $3 \mathrm{~L}+0 \mathrm{~S}$ & & $5-\mathrm{B}$ & $1 \mathrm{~L}+(n-1) \mathrm{S}$ \\
\hline \multirow{3}{*}{2} & $2-\mathrm{A}$ & $0 \mathrm{~L}+2 \mathrm{~S}$ & \multirow{5}{*}{4} & $4-\mathrm{A}$ & $0 \mathrm{~L}+4 \mathrm{~S}$ & & $5-\mathrm{C}$ & $2 \mathrm{~L}+(n-2) \mathrm{S}$ \\
\hline & $2-B$ & $1 \mathrm{~L}+1 \mathrm{~S}$ & & $4-\mathrm{B}$ & $1 \mathrm{~L}+3 \mathrm{~S}$ & & $5-\mathrm{D}$ & $3 \mathrm{~L}+(n-3) \mathrm{S}$ \\
\hline & $2-B$ & $2 \mathrm{~L}+0 \mathrm{~S}$ & & $4-\mathrm{C}$ & $2 \mathrm{~L}+2 \mathrm{~S}$ & & $5-\mathrm{E}$ & $4 \mathrm{~L}+(n-4) \mathrm{S}$ \\
\hline \multirow{2}{*}{3} & $3-\mathrm{A}$ & $0 \mathrm{~L}+3 \mathrm{~S}$ & & $4-\mathrm{D}$ & $3 \mathrm{~L}+1 \mathrm{~S}$ & & $5-\mathrm{F}$ & $5 \mathrm{~L}+(n-5) \mathrm{S}$ \\
\hline & $3-\mathrm{B}$ & $1 \mathrm{~L}+2 \mathrm{~S}$ & & $4-\mathrm{E}$ & $4 \mathrm{~L}+0 \mathrm{~S}$ & & $5-\mathrm{G}$ & $\mathrm{xL}+(n-\mathrm{x}) \mathrm{S}$ \\
\hline
\end{tabular}



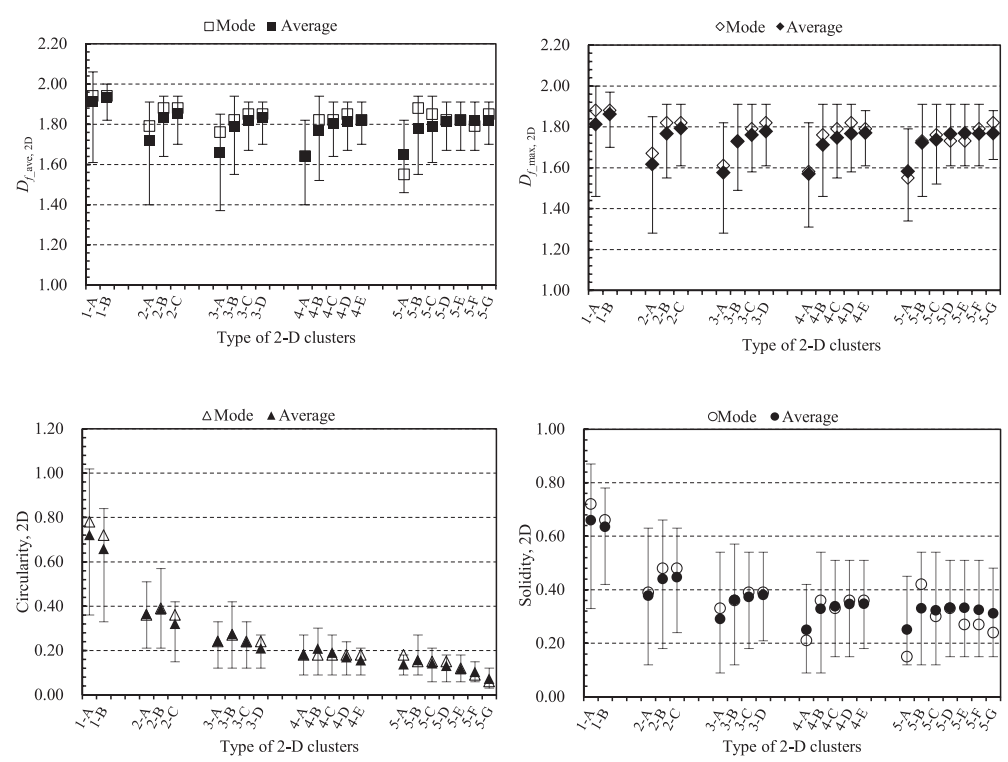

Fig. 14. Characteristics of parameters to describe 2D cluster structure.

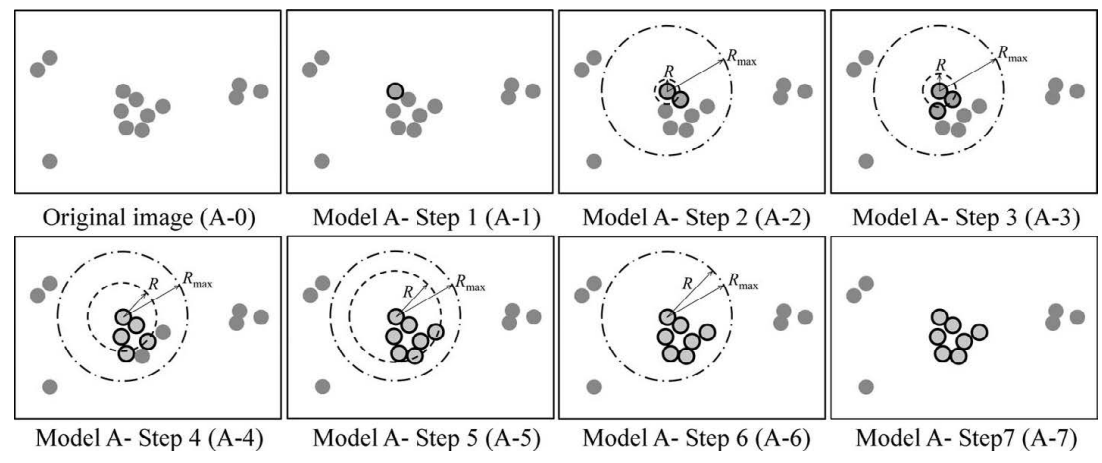

Fig. 15. Steps of distinguishing $2 \mathrm{D}$ clusters (Model A).
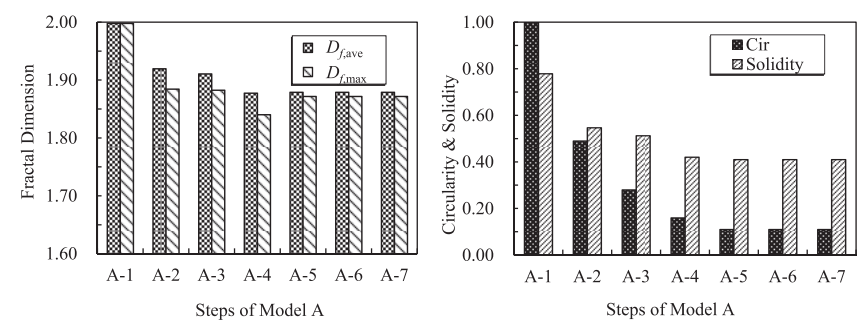

Fig. 16. Change of $2 \mathrm{D}$ parameters with steps in Model A.

selected particle in step (1). Particles in the range covered by the circle are assumed as a $2 \mathrm{D}$ cluster, which are marked with black edge. The $2 \mathrm{D}$ parameters of the assumed cluster are calculated. (see Fig. 15(A-2)).

(3) The calculated 2D parameters in step (2) are compared with the critical range of these parameters. The critical ranges of each $2 \mathrm{D}$ parameter were set according to the particle number and size included in the assumed clusters, which are shown in Fig. 14.

(4) The selected particles are recognized as one cluster if the calculated 2D parameters are in the critical range. Otherwise, they are not one cluster; the program is stopped and goes to step (1).

(5) The circle of the selected range with radius $R$ increase from step A-3 and more particles are covered (particles with black edge).
(6) Repeat step (3) and (4). A maximum range with radius $R_{\max }$ is set to reduce the computation loads, which is enough large in the present study.

(7) The program stops when the selected range reaches to the maximum diameter $R_{\max }$. (see Fig. $15(\mathrm{~A}-6)$ ), and the cluster is finally distinguished as shown in Fig. 15(A-7).

The Model A above mentioned is the simplest case, in which the clusters are well isolated to others. Figure 17 shows a more complicate case, Model B. The steps from B0 to B-3 in Model B shown in Fig. 17 are the same as these from A-0 to A-3 in Model A. However, the selected range of circle encounters a single particle at step B-6 before it reaches the maximum $R_{\max }$. The parameters of the assumed clusters are out of the critical range, particularly the fractal dimension, which shows a sudden change in Fig. 18(a) at step B-6.

Furthermore, the most complicate case is the Model C, in which the selected range circle encounters a single particle before it covers all the particles in the large cluster. Figure 19 shows the steps to distinguish clusters in Model $\mathrm{C}$. The steps from C-0 to C-3 in Model C shown in Fig. 19 are the same as Model A from A-0 to A-3. Then the selected circle encounters a single particle at step C-4; and the 2D parameters are suddenly changed and out of the critical range, such as shown in the step C-4 in Fig. 20(a). The program broken at step C-4 and the result is shown in step C-5. Then 


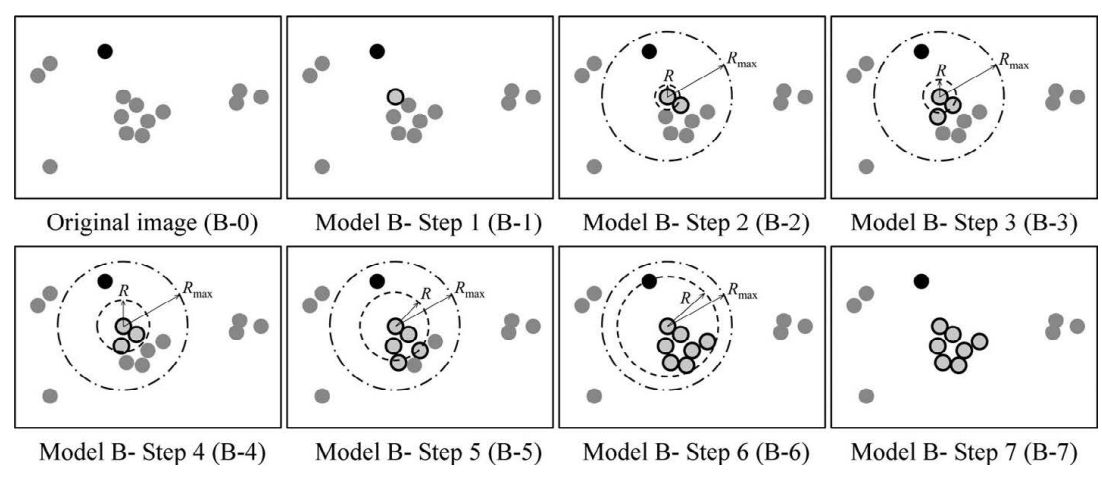

Fig. 17. Steps of distinguishing 2D clusters (Model B).

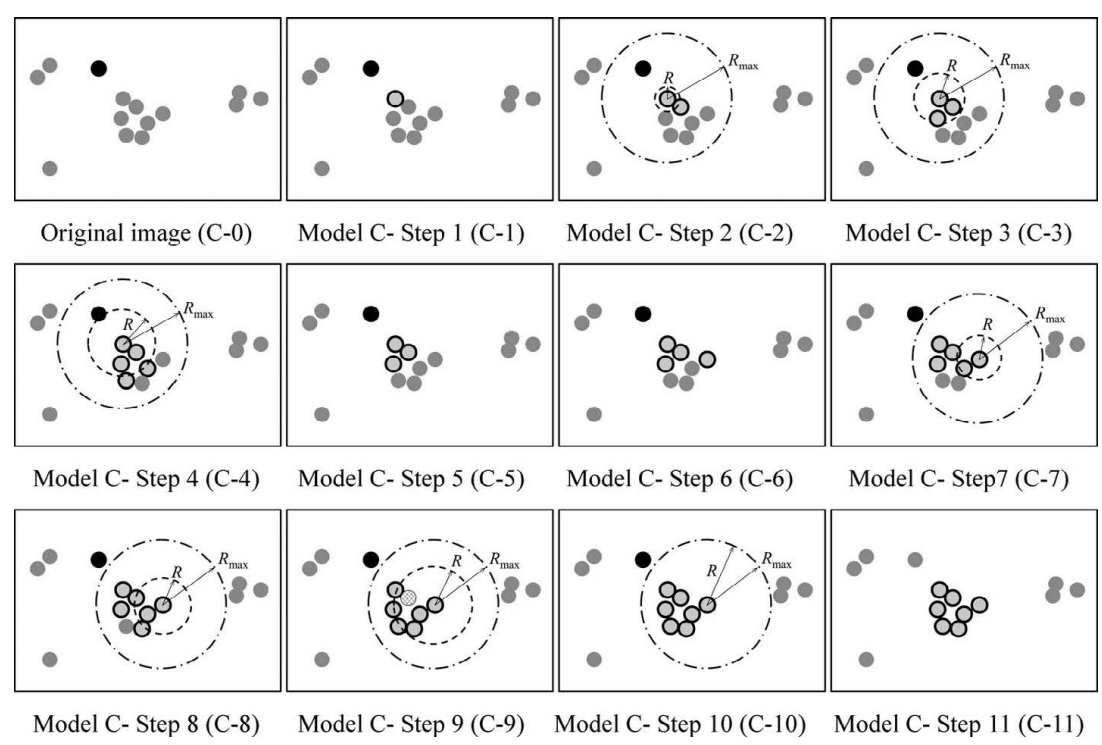

Fig. 19. Steps of distinguishing $2 \mathrm{D}$ clusters (Model C).
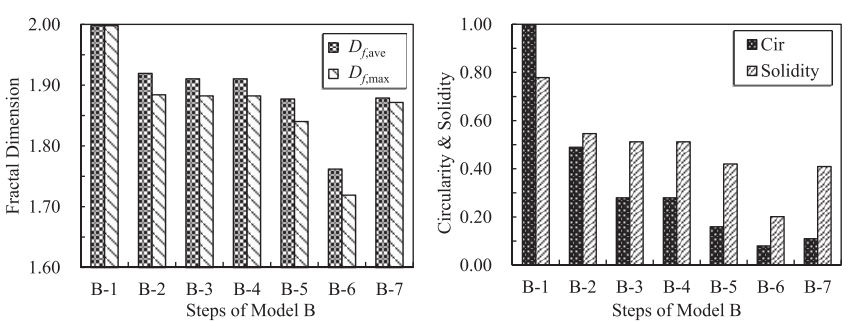

Fig. 18. Change of $2 \mathrm{D}$ parameters with steps in Model B.
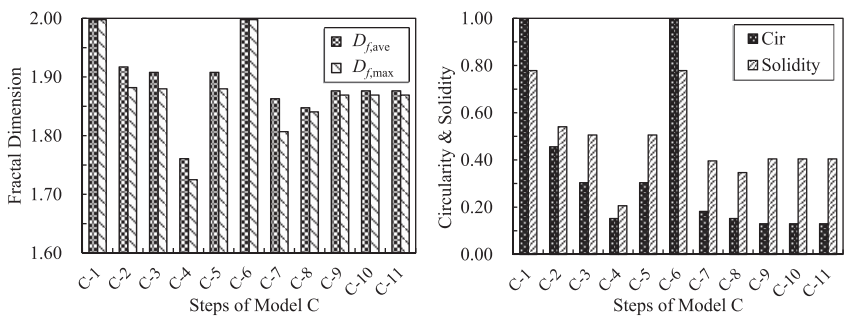

Fig. 20. Change of $2 \mathrm{D}$ parameters with steps in Model C.

a new particle is selected at step C-6 and the former process is repeated from step C-7. The cluster is finally distinguished as shown in step C-11 of Fig. 19.

\subsection{Verification with $3 D$ Information}

The actual 2D cross-sectional X-ray micro-CT images

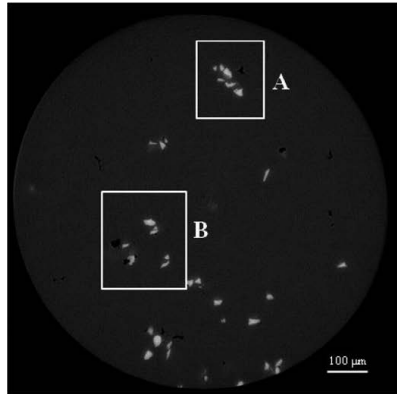

(a) Raw 8-bit micro-CT image.

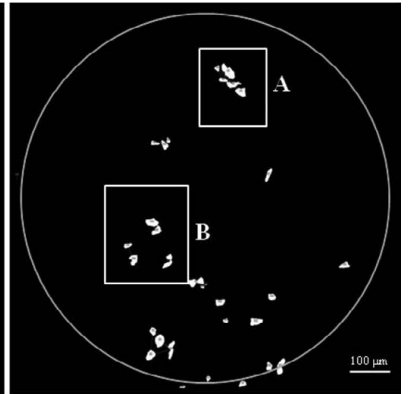

(b) Image processed micro-CT image.
Fig. 21. Raw 8-bit micro-CT image and image processed image.

were processed by the program of DC-2D-3D discussed above. Figure 21(a) shows the raw micro-CT image; and Fig. 21(b) shows the image processed by the program of DC-2D-3D, in which particles belonging to one cluster were connected by straight lines.

Figure 22 shows the examples of the distinguished 2D clusters. The label numbers on the particles are sequence number of a cluster. The particles labeled by the same sequence number belong to one cluster, which are obtained by the program of 3D-Extracter verified using 3D information in image stacks. Figure 22(a) shows a well-distinguished cluster, which is relatively isolated in the whole 2D crosssectional images in Fig. 21. There are three small clusters 


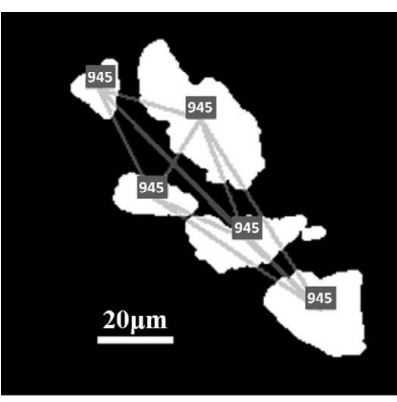

(a)

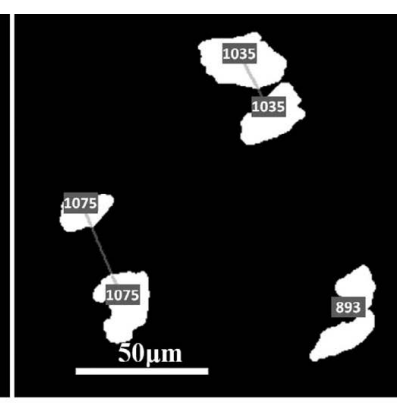

(b)
Fig. 22. Examples of successfully distinguished clusters.

distinguished in Fig. 22(b); these are not distinguished as one whole cluster. It also proves the reliability of the program of DC-2D-3D.

\section{Conclusion}

In this study, $\mathrm{TiB}_{2}$ particles were used to prepare clusters in molten $\mathrm{Al}$ agitated in a two baffled crucible. The samples of $\mathrm{Al}-\mathrm{TiB}_{2}$ were measured by X-ray micro-CT to obtain the $3 \mathrm{D}$ information of $\mathrm{TiB}_{2}$ particles and clusters in the solid $\mathrm{Al}$. Series of cross-sectional X-ray micro-CT images from the samples of $\mathrm{Al}_{-} \mathrm{TiB}_{2}$ were obtained. The characteristics of the 3D particles and clusters were analyzed using self-developed programs. The conclusions of this study are summarized as follows:

(1) A program of 3D Extractor was developed to extract 3D particles and clusters from a series of X-ray micro-CT images of $\mathrm{Al}-\mathrm{TiB}_{2}$ samples. A number of $\mathrm{TiB}_{2}$ clusters were extracted by the program of $3 \mathrm{D}$ Extractor. Various parameters were defined to describe the characteristics of $3 \mathrm{D}$ particles and clusters and their 2D cross-sections.

(2) The 3D cluster structure was not affected by the agitation time and speed. Whereas the particle number included in clusters affects greatly on their structure. The specific surface and solidity decrease greatly with particle number, which indicate the clusters becomes looser with the particle number increase.

(3) There was no apparent changes in $D_{f \text {,ave,3D }}$ and

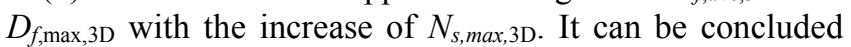
roughly that the representative value of the fractal dimension of the $\mathrm{TiB}_{2}$ clusters formed in molten $\mathrm{Al}$ is selected as 2.7 .

(4) The representative value of the 2D fractal dimension of $\mathrm{TiB}_{2}$ clusters is selected as 1.8 . The relation between the $3 \mathrm{D}$ and $2 \mathrm{D}$ fractal dimensions for $\mathrm{TiB}_{2}$ clusters is corresponding to that between $3 \mathrm{D}$ and $2 \mathrm{D}$ for spheres. The $2 \mathrm{D}$ clusters were successfully distinguished by the program of DC-2D-3D according to the 2D parameters of cluster slices obtained from actual 3D clusters.

\section{Acknowledgements}

This work was partly supported by Grant-in-Aid for Scientific Research (A) (No. 22246097) which is provided by Japan Society for the Promotion of Science (JSPS). Project number and beam line of SPring- 8 are 2011B1395 and BL20XU. The authors wish to express thanks to Dr. A. Takeuchi and Dr. Y. Suzuki for their help during the operation of the X-ray micro CT at SPring-8, Dr. M. Iguchi of Nihon Ceratec Co., Ltd. for providing the Al-TiB 2 MMC, and Dr. T. Iizuka of Isuzu Advanced Engineering Center Ltd. for the help to disperse particle in the Al-7wt.\% $\%$ alloy.

\section{REFERENCE}

1) P. G. Saffman and J. S. Turner: J. Fluid Mech., 1 (1956), 16.

2) K. Higashitani, R. Ogawa, G. Hosokawa and Y. Matsuno: J. Chem. Eng. Jpn., 15 (1982), 299.

3) K. Higashitani, K. Yamauchi, Y. Matsuno and G. Hosokawa: J. Chem. Eng. Jpn., 16 (1983), 299.

4) S. Taniguchi, A. Kikuchi, T. Ise and N. Shoji: ISIJ Int., 36 (1996), S117.

5) T. Nakaoka, S. Taniguchi, K. Matsumoto and S. T. Johansen: ISIJ Int., 41 (2001), 1103.

6) H. Arai, K. Matsumoto, S. Shimasaki and S. Taniguchi: ISIJ Int., 49 (2009), 965.

7) K. Falconer: Fractal Geometry, John Wiley \& Sons, West Sussec, England, (2003), 27.

8) S. Hans: Space-Filling Curves, Springer-Verlag, New York, (1994), 156.

9) T. Vicsek: Fractal Growth Phenomena, World Scientific, Singapore, (1992), 10.

10) R. Jullien, M. Kolb and R. Botet: J. Physique Lett., 45 (1984), L211.

11) T. A. Witten and L. M. Sander: Phys. Rev. Lett., 47 (1981), 1400.

12) P. Meakin: Phys. Rev. A, 27 (1983), 604.

13) P. Meakin and H. E. Stanley: Phys. Rev. Lett., 51 (1983), 1457.

14) M. Muthukumar: Phys. Rev. Lett., 50 (1983), 839.

15) H. Tozawa, Y. Kato, K. Sorimachi and T. Nakanishi: ISIJ Int., 39 (1999), 426

16) W.-C. Doo, D.-Y. Kim, S.-C. Kang and K.-W. Yi: ISIJ Int., 47 (2007), 1070.

17) S. R. Forrest and T. A. Witten: J. Phys. A, Math. Gen., 12 (1979), L109.

18) C. Lee and T. A. Kramer: Adv. Colloid. Interface Sci., 112 (2004), 49.

19) M. L. Eggersdorfer, D. Kadau, H. J. Herrmann and S. E. Pratsinis: $J$. Colloid. Interface Sci., 342 (2010), 261.

20) T. O. Mbuya, I. Sinclair, K. A. Soady and P. A. S. Reed: Proc. 13th Int. Conf. on Aluminum Alloys, TMS, Warrendale, PA, (2012), 55.

21) J.-Y. Buffière, E. Maire, P. Cloetens, G. Lormand and R. Fourgères: Acta Mater., 47 (1999), 1613.

22) A. Elmoutaouakkil, G. Fuchs, P. Bergounhon, R. Péres and F. Peyrin: J. Phys. D, Appl. Phys., 36 (2003), A37.

23) M. Gonzalez, G. Dominguez and C. Bathias: J. Compos. Technol. Res., 22 (2000), 45.

24) A. Guvenilir and S. R. Stock: Fatigue Fract. Eng. Mater. Struct., 21 (1998), 439.

25) E. Maire, L. Babout, J.-Y. Buffiere and R. Fougeres: Mater. Sci. Eng. A, 319-321 (2001), 216.

26) L. Salvo, P. Cloetens, E. Maire, S. Zabler, J. J. Blandin, J. Y. Buffière, W. Ludwig, E. Boller, D. Bellet and C. Josserond: Nucl. Instrum. Method. Phys. Res. B, 200 (2003), 273.

27) R. W. Hamilton, M. F. Forste, R. J. Dashwood and P. D. Lee: Scr. Mater., 46 (2002), 25.

28) T. Li, S. Shimasaki, S. Taniguchi, K. Uesugi and S. Narita: Metall. Mater. Trans. B, 44 (2013), 750.

29) R. L. Basmann and D. J. Slottje: J. Am. Stat. Assoc., 55 (1960), 650. 